\section{Relationship between age and injury severity in traffic accidents involving elderly pedestrians}

\author{
Hyog Ho Lee', Jin-Seong Cho², Yong Su Lim², Sung Youl Hyun', \\ Jae-Hyug $\mathrm{Woo}^{2}$, Jae Ho Jang ${ }^{1}$, Hyuk Jun Yang ${ }^{2}$ \\ 'Department of Emergency Medicine, Gachon University Gil Hospital, Incheon, Korea \\ ${ }^{2}$ Department of Emergency Medicine, Gil Medical Center, Gachon University College of Medicine, Incheon, \\ Korea \\ ${ }^{3}$ Department of Traumatology, Gil Medical Center, Gachon University College of Medicine, Incheon, Korea
}

Objective This study aimed to examine whether injury severity differs with respect to age among elderly pedestrians involved in traffic accidents and identify factors affecting injury severity.

Methods Using emergency department-based injury in-depth surveillance data, we analyzed the data of patients aged $\geq 60$ years who were victims of pedestrian traffic accidents during 2011 to 2016. The pedestrians' ages were divided into 5-year age strata beginning at 60 years. In a multivariate analysis, injury severity was classified as severe to critical or mild to moderate.

Results The analysis included 10,449 patients. All age groups had a female predominance, and accidents most frequently occurred during the early morning. Multivariate analyses revealed that compared to the 60 to 64 years group, the odds ratios for incurring a severe injury were 1.18 (95\% confidence interval [Cl], 1.02 to 1.37$)$ for the 65 to 69 years group, $1.42(95 \% \mathrm{Cl}, 1.23$ to 1.64$)$ for the 70 to 74 years group, $1.70(95 \% \mathrm{Cl}, 1.45$ to 1.98$)$ for the 75 to 79 years group, and $1.83(95 \% \mathrm{Cl}, 1.56$ to 2.15$)$ for the $\geq 80$ years group.

Conclusion In this study of emergency department-based data, we found that injury severity increased with age among elderly victims of traffic accidents. Furthermore, injury severity varied with respect to sex, time and location of the accident, and type of vehicle involved. Therefore, measures intended to reduce and prevent traffic accidents involving elderly pedestrians should consider these findings.

Keywords Aged; Pedestrians; Accidents; Traffic; Risk factors
eISSN: 2383-4625

Received: 29 June 2018

Revised: 24 August 2018

Accepted: 8 September 2018

Correspondence to: Jin-Seong Cho Department of Emergency Medicine, Gil Medical Center, Gachon University College of Medicine, 783 Namdongdaero, Namdong-gu, Incheon 21556, Korea

E-mail: truecho@gilhospital.com ORCID

http://orcid.org/0000-0001-6762-4692

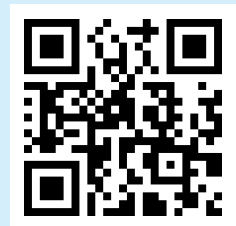

How to cite this article: Lee HH, Cho JS, Lim YS, Hyun SY,

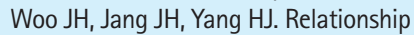
between age and injury severity in traffic accidents involving elderly pedestrians. Clin Exp Emerg Med 2019;6(3):235-241.

What is new in the current study

The severity of pedestrian injuries in traffic accidents increases with age among older adults. Further, we found that injury severity varies in relation to sex, time of accident, type of vehicle involved, and location of accident.
This is an Open Access article distributed under the terms of the Creative Commons Attribution Non-Commercial License (http:// creativecommons.org/licenses/by-nc/4.0/). 


\section{INTRODUCTION}

The incidence of traffic accidents involving elderly individuals is increasing consistently every year as a result of advances in medical technology, extended mean life expectancies, and increased social involvement among the elderly. ${ }^{1}$ Additionally, aging deteriorates physical functions, such as vision and hearing, that may affect the risk of a traffic accident while delaying an individual's response time to risk. ${ }^{2-6}$ Although the number of pedestrian traffic accidents is declining worldwide, the proportion of these accidents that involve the elderly ( $\geq 65$ years) is increasing. According to a statistical analysis of traffic accidents by the Road Traffic Authority, the number of pedestrian traffic accidents involving elderly individuals increased by 1.2-fold from 10,252 in 2013 to 11,978 in 2017, and the number of injury victims from such accidents also increased from 9,413 to 11,224 in the same period?

In addition to the effects of age on an increased risk of pedestrian accidents, elderly victims of traumatic accidents are faced with increased mortality, complication rates, longer hospital stays, and higher medical costs, compared to those in other age groups. ${ }^{8,9}$ Although several studies have investigated the factors affecting traffic accidents involving the elderly population, few studies have obtained data focused on the elderly pedestrians themselves. Moreover, none of the studies have examined differences in injury severity relative to age among elderly pedestrians. Therefore, this study aimed to analyze whether the injury severity consequent to a pedestrian traffic accident differs with regard to age among elderly victims and to identify the factors affecting this severity.

\section{METHODS}

\section{Data collection}

This retrospective cohort study used data from the Emergency Department-based Injury In-depth Surveillance (EDIIS) database, which are collected and managed by the Korea Centers for Disease Prevention and Control. This national prospective database comprises data from injured patients who present to emergency departments (EDs) at 23 currently participating hospitals nationwide. The EDIIS database was developed based on the World Health Organization International Classification of External Causes of Injuries and collects injury-related data to facilitate the development of injury-related national policies. The database contains information collected by ED physicians regarding the patients' demographic characteristics, injury, emergency care records, ED diagnosis, ED treatment, discharge, hospital admission, and outcomes after hospital admission. The collected data were super- vised and corrected by an emergency physician and a skilled research coordinator who had completed a relevant education program for overseeing the EDIIS prior to participating in the project, and entered in the web-based database system by the coordinator. The Quality Control Committee reviewed the database and periodically provided feedback to the EDIIS participants.

\section{Participants}

We used EDIIS data from January 1, 2011 to December 31, 2016. The study participants were patients aged $\geq 60$ years who presented to the ED of one of the participating hospitals after involvement in a pedestrian traffic accident. All pedestrian traffic accidents were included, irrespective of the type of vehicle involved. Patients who were suspected to have visited the ED after visiting another hospital and who did not present directly to the ED immediately after the accident were excluded from the study. Patients whose data lacked important information for the study, such as injury severity, were also excluded.

\section{Definition of variables}

For each patient, sex, age, time of injury, means of hospital admission, and other information were collected. The patients' ages were subdivided in 5-year units into five strata $(60-64,65-69$, $70-74,75-79$, and $\geq 80$ years) to examine differences of injury severity relative to age. The time of injury was classified as daytime (08:00-16:00), evening-time (16:00-24:00), and night-time (00:00-08:00), and the means of hospital transportation was classified as the use or disuse of public ambulance. The indicated drinking status was based on speculation. The types of involved vehicles included a bicycle, motorcycle, car, and others. The type of road on which the traffic accident occurred was classified as a general road, sidewalk, alley, and unknown. The outcomes of ED treatment (e.g., discharge, transfer, admission, expire) and mortality were surveyed as indicators of patient prognosis.

Injury severity was assessed using the excess mortality ratioadjusted injury severity score (EMR-ISS). This system was designed to assess injury severity according to the International Statistical Classification of Diseases and Related Health Problems, 10th revision (ICD-10). Using this system, the excess mortality ratio is computed for all ICD-10 codes in the Korean National Injury Database, which allows classification of the ICD-10 codes into five grades $(1-5) ;$ subsequently, the ISS can be calculated by summing the squares of the three highest severity scores. ${ }^{10}$ Injuries can then be classified as mild (1-8), moderate (9-24), severe (25-74), and critical ( $\geq 75$ ) based on the total EMR-ISS score. Here, patients with EMR-ISS mild and moderate injuries were included in the mild to moderate group, while those with EMR-ISS severe and 
critical injuries were included in the severe to critical group. ${ }^{11,12}$

Unlike other studies, we set severity at the time of injury, rather than mortality or the length of hospital, as the outcome variable. We chose this variable with the intent to minimize the effects of the inevitably higher rates of mortality and morbidity among elderly pedestrians, compared to young adults with the same injury.

\section{Statistical analysis}

Differences in the characteristics of elderly pedestrians among the age strata were compared using chi-square tests. To identify the risk factors for severe injuries, covariates with a P-value of $<0.1$ in the univariate analysis, as well as clinically important covariates, were entered in a multivariate logistic regression with backward stepwise elimination. The data were analyzed using IBM SPSS Statistics ver. 22.0 (IBM Corp., Armonk, NY, USA).

\section{Ethics statement}

The present study was reviewed and approved by the appropriate institutional review board (GCIRB2016-242). The institutional review board waived the requirement for obtaining a signed informed consent.

\section{RESULTS}

The records of 13,364 patients aged $\geq 60$ years who presented at an ED after involvement in a pedestrian traffic accident were examined. After applying the exclusion criteria, 10,449 patients were included in the final analysis (Fig. 1). A total of 4,048 patients were male (38.7\%), and the proportion of female subjects was significantly higher in all age groups $(P<0.001)$. Regarding the time of injury, $44.5 \%$ of all injuries occurred during the nighttime, and night-time accidents were the most frequent in all age groups except for the 60 to 64 years group $(P<0.001)$. The per- centage of patients with suspected alcohol intoxication was $9.3 \%$ in the 60 to 64 years group but decreased to $1.1 \%$ in the $\geq 80$ years group $(\mathrm{P}<0.001)$. Most accidents involved cars, irrespective of age group, whereas the type of vehicle was unknown in $21.6 \%$ of cases. A majority of all accidents occurred on regular roads $(n=6,271 ; 60 \%)$, irrespective of age group. When injury severity was classified according to the EMR-ISS, 2,389 (22.9\%) of cases were classified as severe to critical, while 8,060 (77.14\%) were classified as mild to moderate. However, the overall distribution of injury severity differed significantly among the age groups $(P<0.001)$. After presenting to the ED, 5,667 (54.2\%) were discharged after treatment, 3,325 (31.8\%) were hospitalized, and $525(5.0 \%)$ died in the ED. Missing data were not shown in the table because both the onset time of injury were $21(0.2 \%)$ and the ED outcome were $8(0.08 \%)$ (Table 1).

The largest and smallest proportions of patients were in the 60 to $64(24.5 \%)$ and $\geq 80$ years groups $(14.3 \%)$, and this difference was statistically significant (Table 2). Regarding patient distribution by year, the percentage of patients aged 65 to 69 years remained stable at 24.4\% in 2011 and 24.2\% in 2016, whereas the percentage of patients aged $\geq 80$ years increased significantly from 12.2\% in 2011 to $15.3 \%$ in $2016(P<0.001)$.

To identify risk factors for severe injury, a multivariate analysis that included age, sex, time of injury, means of ED admission, type of vehicle involved, and type of road was performed (Table 3). After setting the 60 to 64 years group as the reference, the odds ratios for incurring a severe injury were 1.18 in the 65 to 69 years group (95\% confidence interval [Cl], 1.02 to 1.37$), 1.42$ in the 70 to 74 years group $(95 \% \mathrm{Cl}, 1.23$ to 1.64$), 1.70$ in the 75 to 79 years group (95\% Cl, 1.45 to 1.98 ), and 1.83 in the $\geq 80$ years group (95\% Cl, 1.56 to 2.15). Compared to the 65 to 69 years group, the odds ratios for incurring a severe injury were 1.2 in the 70 to 74 years group ( $95 \% \mathrm{Cl}, 1.04$ to 1.40$), 1.43$ in the 75 to 79 years group (95\% Cl, 1.23 to 1.67$)$, and 1.54 in the $\geq 80$ years group

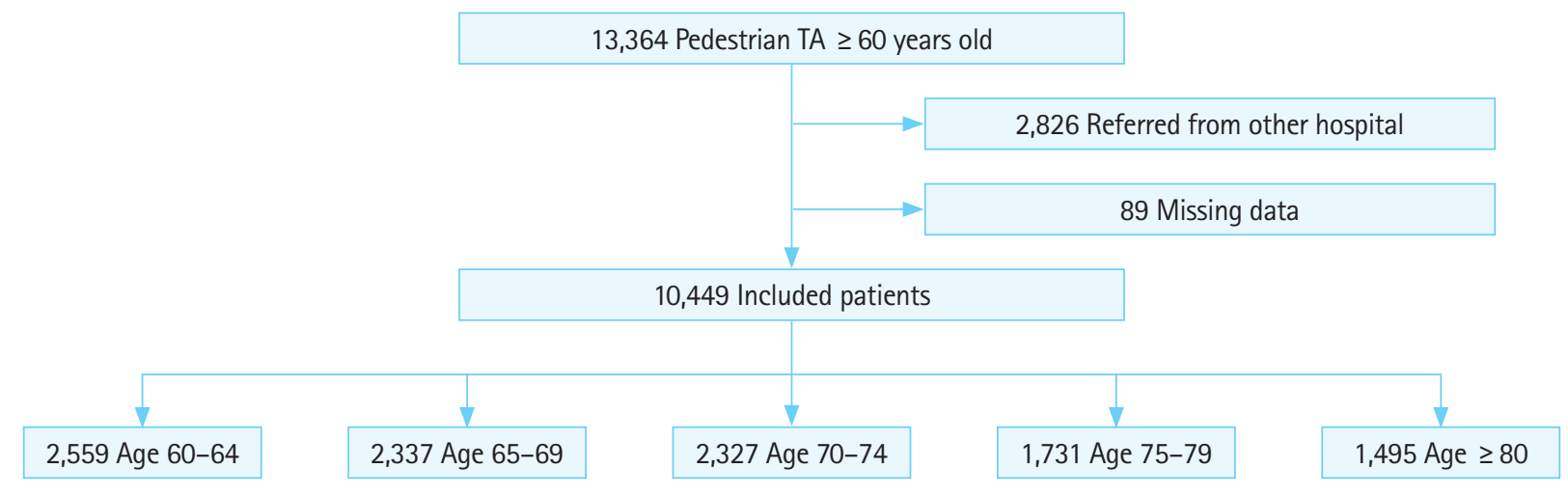

Fig. 1. Flow chart of the study patients. TA, traffic accident. 
Table 1. Baseline characteristics of elderly pedestrians involved in traffic accidents

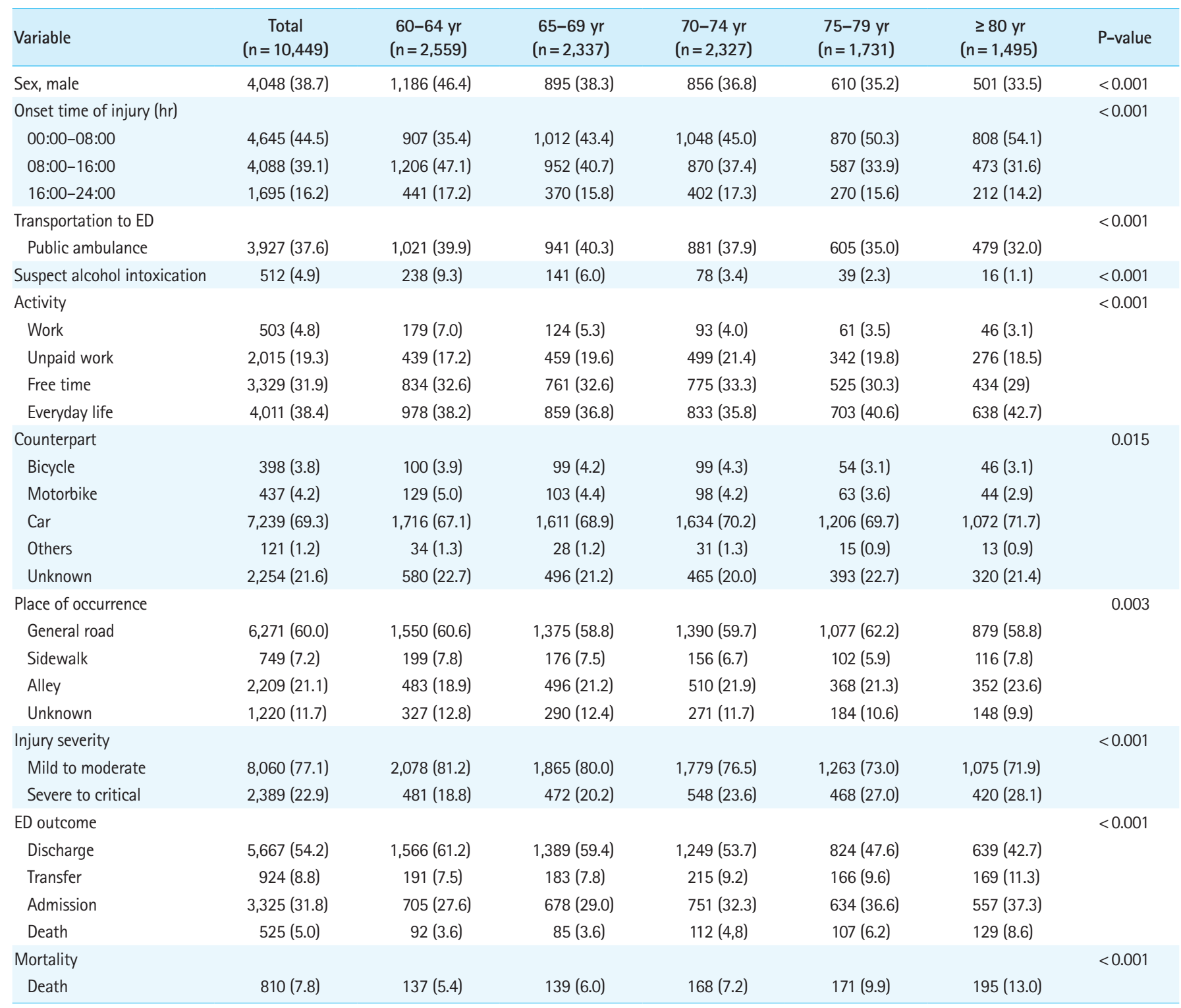

Values are presented as number (\%).

$E D$, emergency department.

Table 2. Patient distribution by year

\begin{tabular}{rrrrrr}
\hline Year & \multicolumn{1}{c}{$60-64 \mathrm{yr}$} & \multicolumn{1}{c}{$65-69 \mathrm{yr}$} & \multicolumn{1}{c}{$70-74 \mathrm{yr}$} & \multicolumn{1}{c}{$75-79 \mathrm{yr}$} & \multicolumn{1}{c}{$\geq 80 \mathrm{yr}$} \\
\hline 2011 & $342(25.1)$ & $333(24.4)$ & $295(21.6)$ & $226(16.6)$ & $166(12.2)$ \\
2012 & $400(24.9)$ & $388(24.1)$ & $403(25.1)$ & $217(13.5)$ & $201(12.5)$ \\
2013 & $417(24.8)$ & $374(22.3)$ & $392(23.3)$ & $251(14.9)$ & $246(14.6)$ \\
2014 & $421(24.5)$ & $361(21.0)$ & $389(22.6)$ & $285(16.6)$ & $262(15.3)$ \\
2015 & $453(23.9)$ & $419(22.1)$ & $392(20.7)$ & $346(18.2)$ & $287(15.1)$ \\
2016 & $526(24.2)$ & $461(21.2)$ & $452(20.8)$ & $404(18.6)$ & $333(15.3)$ \\
Total & $2,559(24.5)$ & $2,336(22.4)$ & $2,323(22.2)$ & $1,731(16.6)$ & $1,495(14.3)$
\end{tabular}

Values are presented as number (\%).

(95\% Cl, 1.31 to 1.81$)$. When age was set as a continuous variable rather than stratified into 5-year groups, the odds ratio for incurring a severe injury with every 1-year increase in age was $1.03(95 \% \mathrm{Cl}, 1.02$ to 1.04$)$.

The odds ratio for incurring a severe injury in accidents involving a car relative to accidents involving a bicycle was 1.67 (95\% $\mathrm{Cl}, 1.64$ to 2.79). Furthermore, the odds ratio for incurring a severe injury in accidents occurring on general road relative to accidents occurring on sidewalk was 1.85 (95\% Cl, 1.49 to 2.30).

\section{DISCUSSION}

In our study of pedestrian traffic accidents among the elderly, we observed a significantly higher risk of incurring a severe injury in 
Table 3. Risk factors for severe injury in univariate and multivariate analyses

\begin{tabular}{|c|c|c|c|c|c|}
\hline \multirow{2}{*}{ Variable } & & \multicolumn{2}{|c|}{ Unadjusted } & \multicolumn{2}{|c|}{ Adjusted } \\
\hline & & Odds ratio & $95 \% \mathrm{Cl}$ & Odds ratio & $95 \% \mathrm{Cl}$ \\
\hline \multirow[t]{6}{*}{ Age (yr) } & $60-64$ & Reference & & Reference & \\
\hline & $65-69$ & 1.09 & $0.95-1.26$ & 1.18 & $1.02-1.37$ \\
\hline & $70-74$ & 1.33 & $1.16-1.53$ & 1.42 & $1.23-1.64$ \\
\hline & $75-79$ & 1.60 & $1.38-1.85$ & 1.70 & $1.45-1.98$ \\
\hline & $\geq 80$ & 1.69 & $1.45-1.96$ & 1.83 & $1.56-2.15$ \\
\hline & Continuous & 1.03 & $1.02-1.03$ & 1.03 & $1.02-1.04$ \\
\hline Sex & Male & 1.22 & $1.12-1.34$ & 1.23 & $1.11-1.36$ \\
\hline \multirow[t]{3}{*}{ TA onset time } & 08:00-16:00 & Reference & & Reference & \\
\hline & $16: 00-24: 00$ & 1.43 & $1.29-1.59$ & 1.43 & $1.28-1.60$ \\
\hline & 00:00-08:00 & 2.13 & $1.88-2.42$ & 1.98 & $1.73-2.27$ \\
\hline Public ambulance & Yes & 4.51 & $4.00-5.09$ & 4.21 & $3.72-4.76$ \\
\hline Alcohol intake & Yes & 1.83 & $1.51-2.21$ & & \\
\hline \multirow[t]{4}{*}{ Vehicle } & Bicycle & Reference & & Reference & \\
\hline & Motorbike & 2.27 & $1.55-3.32$ & 1.42 & $1.62-2.48$ \\
\hline & Car & 2.33 & $1.71-3.18$ & 1.67 & $1.64-2.79$ \\
\hline & Unknown & 2.36 & $1.71-3.26$ & 1.62 & $1.15-2.27$ \\
\hline \multirow[t]{4}{*}{ Place of occurrence } & Sidewalk & Reference & & Reference & \\
\hline & General road & 2.12 & $1.73-2.61$ & 1.85 & $1.49-2.30$ \\
\hline & Alley & 0.88 & $0.69-1.11$ & 0.88 & $0.69-1.12$ \\
\hline & Unknown & 1.02 & $0.79-1.30$ & 1.05 & $0.81-1.36$ \\
\hline
\end{tabular}

$\mathrm{Cl}$, confidence interval; TA, traffic accident.

all age groups relative to the 60 to 64 years group. A previous study reported a higher mean ISS and mortality among pedestrian victims of motorcycle accidents aged $\geq 60$ years, compared to victims aged $<60$ years. ${ }^{13}$ However, that study limited pedestrian traffic accidents to those involving motorcycles, did not subdivide age groups, and did not perform a multivariate analysis. Another study reported that mortality increases with age in pedestrian traffic accidents, consistent with our findings. However, that study stratified age into 10-year intervals and did not consider injury-related factors, such as the type of vehicle involved and location of accidents.

In South Korea, the incidence of traffic accidents in the elderly population ( $\geq 60$ years) is higher among men, with a rate of 11.74 / 1,000 persons per year versus $7.65 / 1,000$ persons per year for women. ${ }^{14}$ Although the incidence of pedestrian traffic accidents was higher among women (61.3\%) in our study, men exhibited more severe injury (odds ratio, 1.23; $95 \% \mathrm{Cl}, 1.11$ to 1.35 ). Furthermore, we observe a trend in which the proportion of female victims increased with age, which was significant given the increasing life expectancy of Korean women over the past decade. ${ }^{15}$ Furthermore, one study reported that men tend to violate traffic regulations more frequently than do women. ${ }^{16}$

The finding that a majority of injuries occurred early in the morning (00:00-08:00) can be attributed to an earlier waking time among the elderly and, consequently, earlier activity. Moreover, traffic conditions during these hours are more amenable to speeding and other traffic law violations, while impaired visibility, inclement weather, and various other factors may also contribute to the involvement of elderly in more severe accidents during this time period. ${ }^{17,18}$ Our observation of more severe injury early in the morning relative to the day-time hours (08:00-16:00) was consistent with the findings of another study in which the severity of injury from traffic accidents was highest at night-time among all pedestrians. ${ }^{19}$

In our analysis, as in other studies, the rate of alcohol consumption was higher among younger victims of pedestrian traffic accidents. ${ }^{20}$ However, although the incidence of severe injury after alcohol consumption was statistically significant in the univariate analysis, this factor did not remain significant in a multivariate analysis using backward elimination and was thus excluded from the final analysis. Accordingly, we determined that alcohol consumption did not have a major effect on the incidence of severe injuries resulting from pedestrian traffic accidents.

Regarding the type of vehicle, the majority of pedestrian traffic accidents in our study involved a car, and the resulting injuries were more severe than those resulting from accidents involving a bicycle. In addition, the type of vehicle involved was unknown in $21.6 \%$ of pedestrian traffic accidents, and these cases were also 
associated with more severe injury, compared to the injuries incurred during accidents involving a bicycle. The high percentage of unknown vehicles may be attributable to the difficulty of obtaining such information from patients who present to the ED while drunk or with altered consciousness.

Some strengths of our study include the use of ED-based data, a multivariate analysis including various injury-related factors (e.g., time and location of accident, type of vehicle involved), and the subdivision of age groups in 5-year strata. The results enable the determination of the age at which the incidence of severe injuries resulting from traffic accidents begins to increase among elderly pedestrians and the identification of individual risk factors. This information can be used to propose effective preventive measures.

However, this study also had a few limitations. First, although underlying diseases, as well as age, may increase injury severity among elderly patients, this study could not include comorbidities as a variable in the multivariate analysis because these data were only available from seven of 23 participating hospitals (missing rate, $60 \%$ to $70 \%$ ). However, we believe that the effects of age partially reflect the effects of underlying diseases. Second, this was a cross-sectional rather than a population-based study. Nevertheless, this study is meaningful because it is based on a large database of information collected from EDs at 23 hospitals during a 6-year period. Third, most participating hospitals are tertiary institutions. Accordingly, patients included in our study may have been transferred to these hospitals, which may have led to an overestimation of injury severity. To eliminate this possibility, we excluded patients who had been transported to the ED via private ambulances under the assumption that these patients were transferred from another hospital. We admit that this may have been inaccurate. Finally, we used the EMR-ISS, rather than the widely used ISS, to assess injury severity because entering the ISS data for all patients at each hospital, an amount of data equivalent to approximately 20,000 to 50,000 pieces of data annually, is a major obstacle. Additionally, it is difficult to control accuracy during this process. Accordingly, we used the EMR-ISS because this system indicates injury severity based on the ICD-10 diagnostic code, an item included in the survey.

In conclusion, the findings of this study based on ED data confirmed that injury severity increased with age among elderly victims of pedestrian traffic accidents. Moreover, we found that the injury severity differed with regard to sex, timing of the accident, type of vehicle involved, and location of the accident. We recommend that preventive measures intended to reduce the number of pedestrian traffic accidents involving elderly people should be developed in consideration of these findings.

\section{CONFLICT OF INTEREST}

No potential conflict of interest relevant to this article was reported.

\section{ACKNOWLEDGMENTS}

The authors are grateful to the Korea Center for Disease Control and Prevention for providing data support for the Emergency Department-based Injury In-depth Surveillance.

\section{REFERENCES}

1. Ahn HC, Seo JY, Chung JB, et al. Clinical review in geriatric trauma patients. J Korean Soc Emerg Med 2002;13:49-54.

2. Klein R. Age-related eye disease, visual impairment, and driving in the elderly. Hum Factors 1991;33:521-5.

3. Mori Y, Mizohata M. Characteristics of older road users and their effect on road safety. Accid Anal Prev 1995;27:391-404.

4. Wong TW. Road traffic accidents in the elderly: a major cause of morbidity and mortality. Ann Acad Med Singapore 1987; 16:101-5.

5. Karceski S, Gold CA. Patient page: driving after a stroke. Neurology 2011;76:e35-8.

6. Gordon-Salant $\mathrm{S}$. Hearing loss and aging: new research findings and clinical implications. J Rehabil Res Dev 2005;42(4 Suppl 2):9-24.

7. Traffic Accident Analysis System. Elderly pedestrian accidents [Internet]. Wonju: KoROAD; 2018 [cited 2018 Apr 28]. Available from: http://taas.koroad.or.kr/sta/acs/gus/selectOdsnPdstrnTfcacd.do?menuld = WEB_KMP_OVT_MVT_TAS_PPT.

8. Champion HR, Copes WS, Buyer D, Flanagan ME, Bain L, Sacco WJ. Major trauma in geriatric patients. Am J Public Health 1989;79:1278-82.

9. Finelli $F C$, Jonsson J, Champion HR, Morelli S, Fouty WJ. A case control study for major trauma in geriatric patients. J Trauma 1989;29:541-8.

10. Kim J, Shin SD, Im TH, et al. Development and validation of the excess mortality ratio-adjusted injury severity score using the international classification of diseases 10th edition. Acad Emerg Med 2009;16:454-64.

11. Baker SP, O'Neill B, Haddon W Jr, Long WB. The injury severity score: a method for describing patients with multiple injuries and evaluating emergency care. J Trauma 1974;14:187-96.

12. Stephenson S, Henley G, Harrison JE, Langley JD. Diagnosis based injury severity scaling: investigation of a method using Australian and New Zealand hospitalisations. Inj Prev 2004; 
10:379-83.

13. Siram SM, Sonaike V, Bolorunduro OB, et al. Does the pattern of injury in elderly pedestrian trauma mirror that of the younger pedestrian? J Surg Res 2011;167:14-8.

14. Hong $K_{\text {, Lee } K M}$, Jang SN. Incidence and related factors of traffic accidents among the older population in a rapidly aging society. Arch Gerontol Geriatr 2015;60:471-7.

15. Kontis V, Bennett JE, Mathers CD, Li G, Foreman K, Ezzati M. Future life expectancy in 35 industrialised countries: projections with a Bayesian model ensemble. Lancet 2017;389:132335.

16. Caleo S. Are organizational justice rules gendered? Reactions to men's and women's justice violations. J Appl Psychol 2016;
101:1422-35.

17. Breen C. CARDI focus on sleep and older people [Internet]. Belfast: Centre for Ageing Research and Development in Ireland; 2014 [cited 2018 Jun 29]. Available from: http://www.cardi. ie/publications/cardifocusonsleepandolderpeople.

18. Owsley C. Aging and vision. Vision Res 2011;51:1610-22.

19. Rifaat SM, Chin HC. Accident severity analysis using ordered probit model. J Adv Transp 2007;41:91-114.

20. Meng Y, Holmes J, Hill-McManus D, Brennan A, Meier PS. Trend analysis and modelling of gender-specific age, period and birth cohort effects on alcohol abstention and consumption level for drinkers in Great Britain using the General Lifestyle Survey 1984-2009. Addiction 2014;109:206-15. 